\title{
The composition of Libraries
}

The following tables show the compositions and their corresponding luminance of five libraries (=generations). The whole population (54 random compositions) in each generation was listed up in an order of decreasing luminance. Two members copied to the next generation by elitism were marked by the bold font. There was no significant difference in luminance of these copied members between former and later generations throughout the whole process, which validates the consistency of our experimental system involving high throughput synthesis and characterization. The composition of the best sample in the fifth generation was marked by the red bold font. This composition can be converted to $\left(\mathrm{Na}_{0.92} \mathrm{Li}_{0.08}\right)\left(\mathrm{Y}_{0.8} \mathrm{Gd}_{0.2}\right) \mathrm{TiO}_{4}: 0.2 \mathrm{Eu}^{3+}$ by neglecting the parameter of excessive alkali metals ( $20 \%$ excess in this case). In fact, even though the parameter of excessive alkali metals was involved in the GACC process, we did not mention this parameter in the description of final composition because it is customary to adopt an excessive amount of alkali metals to compensate for volatilization and also because the final product was proven to be $\left(\mathrm{Na}_{0.92} \mathrm{Li}_{0.08}\right)\left(\mathrm{Y}_{0.8} \mathrm{Gd}_{0.2}\right) \mathrm{TiO}_{4}$ : $\mathrm{Eu}^{3+}$ by structural analyses.

\section{$1^{\text {st }}$ Generation}

\begin{tabular}{|r|r|r|r|c|c|c|c|c|}
\hline $\mathrm{Li}$ & $\mathrm{Na}$ & $\mathrm{K}$ & $\mathrm{Y}$ & $\mathrm{Gd}$ & $\mathrm{La}$ & $\mathrm{Eu}$ & $\mathrm{Ti}$ & Luminance \\
\hline 0.266667 & 0 & 0.266667 & 0.194444 & 0.083333 & 0 & 0.055556 & 0.333333 & 76.16338 \\
0.457143 & 0 & 0 & 0.012422 & 0.062112 & 0.124224 & 0.086957 & 0.428571 & 74.86562 \\
0 & 0.933333 & 0 & 0.107143 & 0 & 0.107143 & 0.119048 & 0.333333 & 72.13138 \\
0 & 0.311111 & 0.088889 & 0.079365 & 0.031746 & 0.079365 & 0.142857 & 0.333333 & 69.59097 \\
0.65 & 0.108333 & 0.108333 & 0 & 0.083333 & 0.166667 & 0.083333 & 0.333333 & 67.54859 \\
0.2 & 0.2 & 0 & 0.111111 & 0.194444 & 0 & 0.027778 & 0.333333 & 66.52014 \\
0 & 0 & 0.514286 & 0 & 0.204082 & 0 & 0.081633 & 0.428571 & 65.86415 \\
0 & 0 & 0.342857 & 0.175824 & 0 & 0.043956 & 0.065934 & 0.428571 & 65.82766 \\
0.0875 & 0.116667 & 0.2625 & 0 & 0.133333 & 0.083333 & 0.116667 & 0.333333 & 65.63245 \\
0.333333 & 0.266667 & 0.133333 & 0.140351 & 0 & 0.122807 & 0.070175 & 0.333333 & 65.21205 \\
0.133333 & 0.666667 & 0 & 0 & 0 & 0.196078 & 0.137255 & 0.333333 & 64.36816 \\
0.062338 & 0.280519 & 0 & 0.030075 & 0.105263 & 0.06015 & 0.090226 & 0.428571 & 63.90662 \\
0 & 0.095238 & 0.571429 & 0.05 & 0.166667 & 0 & 0.116667 & 0.333333 & 63.71057 \\
0.366667 & 0.366667 & 0 & 0 & 0 & 0.242424 & 0.090909 & 0.333333 & 63.54499 \\
0 & 0.466667 & 0 & 0.185185 & 0 & 0 & 0.148148 & 0.333333 & 62.51127
\end{tabular}




\begin{tabular}{|c|c|c|c|c|c|c|c|c|}
\hline 0.228571 & 0 & 0.114286 & 0 & 0.204082 & 0 & 0.081633 & 0.428571 & 60.35012 \\
\hline 0 & 0.3 & 0.214286 & 0.078818 & 0.049261 & 0.068966 & 0.08867 & 0.428571 & 59.64794 \\
\hline 0.16 & 0.091429 & 0.091429 & 0.05291 & 0.074074 & 0.084656 & 0.074074 & 0.428571 & 59.60853 \\
\hline 0.095238 & 0 & 0.190476 & 0.063492 & 0.079365 & 0.063492 & 0.079365 & 0.428571 & 58.864 \\
\hline 0 & 0 & 0.733333 & 0.078431 & 0.058824 & 0 & 0.196078 & 0.333333 & 57.3843 \\
\hline 0.16 & 0.091429 & 0.091429 & 0.05291 & 0.074074 & 0.084656 & 0.074074 & 0.428571 & 54.48128 \\
\hline 0.072727 & 0 & 0.327273 & 0 & 0.133333 & 0.111111 & 0.088889 & 0.333333 & 53.89187 \\
\hline 0.177778 & 0.111111 & 0.111111 & 0.061224 & 0.102041 & 0 & 0.122449 & 0.428571 & 53.80262 \\
\hline 0.380952 & 0 & 0.190476 & 0.183673 & 0.061224 & 0 & 0.040816 & 0.428571 & 53.3078 \\
\hline 0.311688 & 0.155844 & 0.103896 & 0.190476 & 0.071429 & 0 & 0.02381 & 0.428571 & 52.18583 \\
\hline 0 & 0 & 0.6 & 0.02381 & 0.166667 & 0 & 0.142857 & 0.333333 & 51.25764 \\
\hline 0.16 & 0.32 & 0.32 & 0 & 0.133333 & 0 & 0.2 & 0.333333 & 51.23906 \\
\hline 0.477551 & 0 & 0.265306 & 0.10084 & 0.016807 & 0 & 0.168067 & 0.428571 & 49.06005 \\
\hline 0 & 0.217778 & 0.248889 & 0 & 0.133333 & 0 & 0.2 & 0.333333 & 48.94658 \\
\hline 0.342857 & 0 & 0 & 0.047619 & 0.142857 & 0.02381 & 0.071429 & 0.428571 & 48.86352 \\
\hline 0.02807 & 0.252632 & 0.252632 & 0.095238 & 0 & 0 & 0.238095 & 0.333333 & 47.48637 \\
\hline 0.116667 & 0 & 0.35 & 0.1 & 0 & 0.166667 & 0.066667 & 0.333333 & 47.0874 \\
\hline 0.52 & 0.222857 & 0 & 0 & 0 & 0.107143 & 0.178571 & 0.428571 & 46.3708 \\
\hline 0.742857 & 0 & 0 & 0.074534 & 0.099379 & 0 & 0.111801 & 0.428571 & 45.49914 \\
\hline 0.4 & 0 & 0 & 0 & 0 & 0.25 & 0.083333 & 0.333333 & 45.38617 \\
\hline 0 & 0.4 & 0.4 & 0 & 0.156863 & 0 & 0.176471 & 0.333333 & 43.76872 \\
\hline 0.3 & 0 & 0.3 & 0.111111 & 0.055556 & 0.125 & 0.041667 & 0.333333 & 43.19052 \\
\hline 0 & 0 & 0.466667 & 0.111111 & 0.027778 & 0.138889 & 0.055556 & 0.333333 & 40.51265 \\
\hline 0 & 0 & 0.666667 & 0 & 0 & 0.148148 & 0.185185 & 0.333333 & 39.24277 \\
\hline 0 & 0 & 0.866667 & 0.111111 & 0.013889 & 0.138889 & 0.069444 & 0.333333 & 38.68927 \\
\hline 0 & 0.102564 & 0.230769 & 0 & 0 & 0.095238 & 0.238095 & 0.333333 & 37.96127 \\
\hline 0 & 0 & 0.333333 & 0 & 0.166667 & 0.15 & 0.016667 & 0.333333 & 37.15427 \\
\hline 0 & 0.155556 & 0.777778 & 0.123457 & 0.111111 & 0.08642 & 0.012346 & 0.333333 & 36.68268 \\
\hline 0.444444 & 0.355556 & 0.133333 & 0.111111 & 0 & 0 & 0.222222 & 0.333333 & 32.42828 \\
\hline 0.4 & 0.4 & 0 & 0.066667 & 0 & 0 & 0.266667 & 0.333333 & 31.66369 \\
\hline 0 & 0.8 & 0 & 0 & 0.111111 & 0 & 0.222222 & 0.333333 & 31.18226 \\
\hline 0 & 0.266667 & 0.066667 & 0 & 0 & 0.055556 & 0.277778 & 0.333333 & 30.70995 \\
\hline
\end{tabular}




\begin{tabular}{|c|c|c|c|c|c|c|c|c|}
\hline 0.4 & 0 & 0 & 0 & 0.117647 & 0.039216 & 0.176471 & 0.333333 & 30.63688 \\
\hline 0.228571 & 0.228571 & 0 & 0.047619 & 0 & 0 & 0.238095 & 0.428571 & 30.58309 \\
\hline 0.342857 & 0 & 0 & 0.152381 & 0.019048 & 0.095238 & 0.019048 & 0.428571 & 29.5034 \\
\hline 0 & 0.678788 & 0.254545 & 0.066667 & 0 & 0 & 0.266667 & 0.333333 & 28.98147 \\
\hline 0.333333 & 0 & 0 & 0.214286 & 0 & 0 & 0.119048 & 0.333333 & 28.79075 \\
\hline 0.8 & 0 & 0 & 0 & 0.111111 & 0.037037 & 0.185185 & 0.333333 & 28.59504 \\
\hline 0 & 0.333333 & 0 & 0 & 0.041667 & 0 & 0.291667 & 0.333333 & 26.91967 \\
\hline
\end{tabular}

$2^{\text {nd }}$ Generation

\begin{tabular}{r|r|r|r|c|c|c|c|r|}
\hline \multicolumn{1}{c|}{$\mathrm{Li}$} & $\mathrm{Na}$ & $\mathrm{K}$ & $\mathrm{Y}$ & $\mathrm{Gd}$ & $\mathrm{La}$ & $\mathrm{Eu}$ & $\mathrm{Ti}$ & Luminance \\
\hline 0.133333 & 0 & 0.266667 & 0.061224 & 0.102041 & 0 & 0.122449 & 0.428571 & 84.02618 \\
0.02807 & 0.252632 & 0.252632 & 0.095238 & 0.142857 & 0 & 0.095238 & 0.333333 & 72.16902 \\
$\mathbf{0 . 4 5 7 1 4}$ & 0 & $\mathbf{0}$ & $\mathbf{0 . 0 1 2 4 2}$ & $\mathbf{0 . 0 6 2 1 1}$ & $\mathbf{0 . 1 2 4 2 2}$ & $\mathbf{0 . 0 8 6 9 6}$ & $\mathbf{0 . 4 2 8 5 7}$ & $\mathbf{7 2 . 0 5 1 6}$ \\
0.577778 & 0.288889 & 0 & 0 & 0.133333 & 0.111111 & 0.088889 & 0.333333 & 71.26265 \\
$\mathbf{0 . 2 6 6 6 7}$ & 0 & $\mathbf{0 . 2 6 6 6 7}$ & $\mathbf{0 . 1 9 4 4 4}$ & $\mathbf{0 . 0 8 3 3 3}$ & $\mathbf{0}$ & $\mathbf{0 . 0 5 5 5 6}$ & $\mathbf{0 . 3 3 3 3 3}$ & $\mathbf{7 0 . 8 4 5 8}$ \\
0.114286 & 0.571429 & 0 & 0.171429 & 0.07619 & 0 & 0.038095 & 0.428571 & 70.79257 \\
0 & 0.35 & 0.25 & 0.133333 & 0.083333 & 0 & 0.116667 & 0.333333 & 69.38272 \\
0 & 0.095238 & 0.571429 & 0.05 & 0.166667 & 0 & 0.116667 & 0.333333 & 68.74266 \\
0 & 0 & 0.342857 & 0.095238 & 0 & 0.071429 & 0.119048 & 0.428571 & 64.94454 \\
0.114286 & 0 & 0.228571 & 0.05291 & 0.074074 & 0.084656 & 0.074074 & 0.428571 & 64.49399 \\
0.366667 & 0.366667 & 0 & 0.083333 & 0.033333 & 0.083333 & 0.133333 & 0.333333 & 63.5501 \\
0 & 0 & 0.866667 & 0.05 & 0.166667 & 0 & 0.116667 & 0.333333 & 62.57879 \\
0 & 0.266667 & 0.07619 & 0.142857 & 0.085714 & 0 & 0.057143 & 0.428571 & 62.5363 \\
0.257143 & 0 & 0.257143 & 0.07619 & 0.066667 & 0.07619 & 0.066667 & 0.428571 & 62.25151 \\
0.195918 & 0.04898 & 0.097959 & 0 & 0.168067 & 0 & 0.117647 & 0.428571 & 62.13912 \\
0.095238 & 0 & 0.190476 & 0.063492 & 0.079365 & 0.063492 & 0.079365 & 0.428571 & 61.76788 \\
0 & 0.081633 & 0.489796 & 0.029557 & 0.098522 & 0.068966 & 0.08867 & 0.428571 & 61.62765 \\
0 & 0.095238 & 0.571429 & 0.052632 & 0.157895 & 0 & 0.122807 & 0.333333 & 61.29024 \\
0.14 & 0.08 & 0.18 & 0 & 0.142857 & 0.119048 & 0.071429 & 0.333333 & 60.64062 \\
0.266667 & 0 & 0.266667 & 0.212121 & 0 & 0 & 0.121212 & 0.333333 & 59.3167 \\
0.02807 & 0.252632 & 0.252632 & 0.035088 & 0 & 0.175439 & 0.122807 & 0.333333 & 58.78649 \\
0.333333 & 0 & 0 & 0 & 0 & 0.238095 & 0.095238 & 0.333333 & 57.92628 \\
0 & 0.095238 & 0.571429 & 0.111111 & 0.013889 & 0.138889 & 0.069444 & 0.333333 & 57.6202 \\
0.366667 & 0.366667 & 0 & 0 & 0 & 0.242424 & 0.090909 & 0.333333 & 57.02874 \\
0.457143 & 0 & 0.285714 & 0.10084 & 0.016807 & 0 & 0.168067 & 0.428571 & 57.02754
\end{tabular}




$\begin{array}{rrrrrrrrr}0.285714 & 0.190476 & 0.095238 & 0.175824 & 0.065934 & 0 & 0.043956 & 0.428571 & 56.8575 \\ 0.075 & 0.1 & 0.225 & 0.175824 & 0 & 0.043956 & 0.065934 & 0.428571 & 56.50088 \\ 0.65 & 0.108333 & 0.108333 & 0 & 0.083333 & 0.166667 & 0.083333 & 0.333333 & 56.36316 \\ 0.16 & 0.091429 & 0.091429 & 0.059524 & 0.083333 & 0.095238 & 0.047619 & 0.428571 & 55.73082 \\ 0.062338 & 0 & 0.280519 & 0 & 0 & 0.107143 & 0.178571 & 0.428571 & 55.31393 \\ 0.630303 & 0.236364 & 0 & 0 & 0 & 0.285714 & 0.047619 & 0.333333 & 55.11716 \\ 0 & 0.35 & 0.05 & 0.185185 & 0 & 0 & 0.148148 & 0.333333 & 55.05916 \\ 0.342857 & 0 & 0.257143 & 0.102564 & 0.051282 & 0.115385 & 0.064103 & 0.333333 & 54.3156 \\ 0 & 0.466667 & 0 & 0.079365 & 0.031746 & 0.079365 & 0.142857 & 0.333333 & 54.27869 \\ 0 & 0 & 0.285714 & 0 & 0.102041 & 0.081633 & 0.102041 & 0.428571 & 53.96238 \\ 0 & 0.311111 & 0.088889 & 0.079365 & 0.031746 & 0.079365 & 0.142857 & 0.333333 & 52.64832 \\ 0.457143 & 0 & 0 & 0.02381 & 0.059524 & 0.119048 & 0.083333 & 0.428571 & 52.48234 \\ 0 & 0 & 0.333333 & 0 & 0.133333 & 0.083333 & 0.116667 & 0.333333 & 52.12362 \\ 0.186667 & 0.106667 & 0.106667 & 0.075758 & 0.060606 & 0.136364 & 0.060606 & 0.333333 & 52.11109 \\ 0.171429 & 0.171429 & 0 & 0.095238 & 0.142857 & 0 & 0.047619 & 0.428571 & 51.98654 \\ 0 & 0.311111 & 0.088889 & 0 & 0 & 0.25 & 0.083333 & 0.333333 & 49.08847 \\ 0.380952 & 0 & 0.190476 & 0.197802 & 0.065934 & 0 & 0.021978 & 0.428571 & 48.61086 \\ 0.207407 & 0.12963 & 0.12963 & 0.1 & 0 & 0.166667 & 0.066667 & 0.333333 & 48.26284 \\ 0.280519 & 0.14026 & 0.093506 & 0.190476 & 0.071429 & 0 & 0.02381 & 0.428571 & 47.15995 \\ 0.2 & 0.2 & 0 & 0.078431 & 0.058824 & 0 & 0.196078 & 0.333333 & 46.75821 \\ 0.444444 & 0 & 0.222222 & 0 & 0 & 0.196078 & 0.137255 & 0.333333 & 46.60047 \\ 0 & 0 & 0.733333 & 0.111111 & 0.194444 & 0 & 0.027778 & 0.333333 & 46.16951 \\ 0.52 & 0.222857 & 0 & 0 & 0 & 0.114286 & 0.171429 & 0.428571 & 45.10304 \\ 0.444444 & 0 & 0.222222 & 0.230769 & 0.076923 & 0 & 0.025641 & 0.333333 & 44.25071 \\ 0.02807 & 0.252632 & 0.252632 & 0.133333 & 0 & 0 & 0.2 & 0.333333 & 42.79013 \\ 0 & 0 & 0.466667 & 0.078431 & 0.019608 & 0.176471 & 0.058824 & 0.333333 & 41.01336 \\ 0 & 0.084848 & 0.848485 & 0.123457 & 0.111111 & 0.08642 & 0.012346 & 0.333333 & 38.33749 \\ 0.444444 & 0 & 0.222222 & 0.115385 & 0.025641 & 0.076923 & 0.115385 & 0.333333 & 38.32814 \\ 0.133333 & 0.666667 & 0 & 0 & 0 & 0 & 0.333333 & 0.333333 & 28.16025\end{array}$

\section{$3^{\text {rd }}$ Generation}

\begin{tabular}{|c|rrrrrrrrrrr|}
\hline $\mathrm{Li}$ & $\mathrm{Na}$ & $\mathrm{K}$ & $\mathrm{Y}$ & $\mathrm{Gd}$ & $\mathrm{La}$ & $\mathrm{Eu}$ & $\mathrm{Ti}$ & Luminance \\
\hline $\mathbf{0 . 1 3 3 3 3}$ & $\mathbf{0}$ & $\mathbf{0 . 2 6 6 6 7}$ & $\mathbf{0 . 0 6 1 2 2}$ & $\mathbf{0 . 1 0 2 0 4}$ & $\mathbf{0}$ & $\mathbf{0 . 1 2 2 4 5}$ & $\mathbf{0 . 4 2 8 5 7}$ & $\mathbf{8 0 . 0 7 1 5}$ \\
0.297744 & 0.330827 & 0 & 0.064935 & 0.025974 & 0.064935 & 0.12987 & 0.428571 & 79.14746 \\
0.213333 & 0 & 0.32 & 0.233333 & 0 & 0 & 0.1 & 0.333333 & 78.56594 \\
$\mathbf{0 . 0 2 8 0 7}$ & $\mathbf{0 . 2 5 2 6 3}$ & $\mathbf{0 . 2 5 2 6 3}$ & $\mathbf{0 . 0 9 5 2 4}$ & $\mathbf{0 . 1 4 2 8 6}$ & $\mathbf{0}$ & $\mathbf{0 . 0 9 5 2 4}$ & $\mathbf{0 . 3 3 3 3 3}$ & $\mathbf{7 2 . 3 6 1 7}$
\end{tabular}




\begin{tabular}{|c|c|c|c|c|c|c|c|c|}
\hline 0 & 0 & 342857 & 0.175824 & 0 & 0.043956 & 0.065934 & 0.428571 & 68.51788 \\
\hline 08 & 10084 & 681 & 9557 & 22 & 56 & 7 & 1 & 68.44965 \\
\hline 0.075 & 0.1 & 225 & 0.095238 & 0 & 0.071429 & 0.119048 & 0.428571 & 7.42126 \\
\hline 0 & 095238 & 0.571429 & .115385 & .025641 & 0.076923 & 0.115385 & 0.333333 & 4.57488 \\
\hline 0.54026 & 202597 & 0 & 0 & 0.064935 & 12987 & 0.090909 & 0.428571 & 4.0618 \\
\hline 0 & 0 & 0.733333 & 0.070175 & 0.122807 & 0.017544 & 0.122807 & 0.333333 & 3.24597 \\
\hline 0 & 0 & 571429 & 0.029557 & 0.098522 & 0.068966 & 0.08867 & 0.428571 & 3.19906 \\
\hline 366667 & 366667 & 0 & 0 & 0 & 0.266667 & 0.066667 & 0.333333 & 2.6292 \\
\hline 577778 & 288889 & 0 & 0 & 0.111111 & 166667 & 0.055556 & 0.333333 & 2.44347 \\
\hline 171429 & 0.171429 & 0 & 0.068027 & 0.081633 & 0 & 0.136054 & 0.428571 & 0.68713 \\
\hline 021053 & 0.189474 & 0.189474 & 0.083333 & 0.0625 & 0 & 0.1875 & 0.333333 & 60.26398 \\
\hline 282353 & 403361 & 0 & 0.171429 & 0.07619 & 0 & 0.038095 & 0.428571 & 0.04464 \\
\hline 533333 & 0 & 0.333333 & 0.2 & .066667 & 0 & 0.066667 & 0.333333 & 59.981 \\
\hline 0 & 0.3 & 0.042857 & 0.15873 & 0 & 0 & 0.126984 & 0.428571 & 59.19736 \\
\hline 266667 & 0.266667 & 0 & 0.083333 & 0.125 & 0 & 0.125 & 0.333333 & 58.98067 \\
\hline 557143 & 092857 & 0.092857 & 0 & 071429 & 857 & 0.071429 & 0.4 & 588 \\
\hline 533333 & 0 & 0 & 0.027778 & 069444 & 38889 & 0.097222 & 0.333333 & 7.91825 \\
\hline 533333 & 0 & 0 & 0.041667 & 0 & 0.25 & 0.041667 & 0.333333 & 57.62604 \\
\hline 0 & 0 & 0.866667 & 0.05 & 0.166667 & 0 & 0.116667 & 0.333333 & 6.12866 \\
\hline 57143 & 146939 & 04 & 0.0 & 13 & 818 & 8966 & 11 & 566 \\
\hline 0.14359 & 184615 & 205128 & 0.133333 & 0 & 0 & 0.2 & 0.333333 & 55.4028 \\
\hline 0.08 & 0.28 & .04 & 0.185185 & 0 & 0 & 0.148148 & 0.333333 & 5.36173 \\
\hline 111111 & 0 & 222222 & 0 & 142857 & 119048 & 0.071429 & 0.333333 & 55.04125 \\
\hline 0 & 685714 & 0 & & & 0 & & & \\
\hline 0.08 & 0.16 & 16 & 0.075758 & .060606 & 0.1 & 606 & 33 & 274 \\
\hline 0.12 & 0.068571 & 0.154 & 0.063492 & 9365 & 492 & 365 & 0 & 648 \\
\hline 0.32 & 0 & 0.213333 & 0.212121 & 0 & 0 & 0.121212 & 0.333333 & 53.36772 \\
\hline 0 & 0 & 0.466667 & 0.222222 & 055556 & 0 & & & \\
\hline 072727 & 0 & 0.327273 & 0 & 0 & & 0.1 & & 5531 \\
\hline 0 & 0.311111 & 0.088889 & 0 & 0 & 769 & 64 & 3 & 27 \\
\hline 0 & 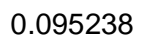 & 0.571429 & & 923 & 0 & 1 & 0.333333 & \\
\hline 228571 & 0 & 0.228571 & 0.111111 & .015873 & 0 & 0.15873 & 0.428571 & 48.13327 \\
\hline 0.2 & 0 & 0.266667 & 0.075758 & 0.060606 & & 0.0 & & 47. \\
\hline 506 & 0.26 & 0 & . & 0 & & & & \\
\hline 0 & 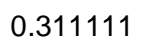 & T.00005 & 0 & 0 & 0.166667 & 7 & & \\
\hline 200 & 85714 & 0 & .197802 & 34 & & 0.021978 & 428571 & 44.337 \\
\hline
\end{tabular}




$\begin{array}{rrrrrrrrr}0.5 & 0 & 0.166667 & 0.111111 & 0.013889 & 0.138889 & 0.069444 & 0.333333 & 43.84146 \\ 0 & 0.866667 & 0 & 0 & 0 & 0.285714 & 0.047619 & 0.333333 & 42.91324 \\ 0.072727 & 0 & 0.327273 & 0 & 0 & 0.125 & 0.208333 & 0.333333 & 42.68616 \\ 0.444444 & 0 & 0.222222 & 0.05 & 0.166667 & 0 & 0.116667 & 0.333333 & 42.09448 \\ 0.444444 & 0 & 0.222222 & 0.130435 & 0.028986 & 0.130435 & 0.043478 & 0.333333 & 42.08793 \\ 0 & 0.042424 & 0.424242 & 0.078431 & 0.019608 & 0.176471 & 0.058824 & 0.333333 & 41.99345 \\ 0.285714 & 0.142857 & 0.142857 & 0.197802 & 0.065934 & 0 & 0.021978 & 0.428571 & 40.44863 \\ 0.488889 & 0 & 0.244444 & 0.083333 & 0.033333 & 0.083333 & 0.133333 & 0.333333 & 40.3419 \\ 0.068571 & 0 & 0.274286 & 0 & 0 & 0.214286 & 0.071429 & 0.428571 & 39.41779 \\ 0 & 0 & 0.866667 & 0.071429 & 0.238095 & 0 & 0.02381 & 0.333333 & 37.57066 \\ 0 & 0.084848 & 0.848485 & 0.123457 & 0.111111 & 0.08642 & 0.012346 & 0.333333 & 33.84998 \\ 0.185714 & 0.557143 & 0 & 0 & 0 & 0.071429 & 0.214286 & 0.428571 & 33.84425 \\ 0.385714 & 0 & 0.128571 & 0.207792 & 0.051948 & 0 & 0.025974 & 0.428571 & 31.64284 \\ 0.380952 & 0 & 0.190476 & 0 & 0 & 0.114286 & 0.171429 & 0.428571 & 31.07718\end{array}$

\section{$4^{\text {th }}$ Generation}

\begin{tabular}{|c|c|c|c|c|c|c|c|c|}
\hline $\mathrm{Li}$ & $\mathrm{Na}$ & $\mathrm{K}$ & $Y$ & $\mathrm{Gd}$ & $\mathrm{La}$ & $\mathrm{Eu}$ & $\mathrm{Ti}$ & Luminance \\
\hline 0 & 0 & 0.4 & 0.095238 & 0 & 0 & 0.190476 & 0.428571 & 81.31835 \\
\hline 0.196491 & 0.252632 & 0.084211 & 0.233333 & 0 & 0 & 0.1 & 0.333333 & 80.86079 \\
\hline 0.13333 & 0 & 0.26667 & 0.06122 & 0.10204 & 0 & 0.12245 & 0.42857 & 80.0016 \\
\hline 0.2 & 0.266667 & 0.2 & 0.05 & 0.166667 & 0 & 0.116667 & 0.333333 & 75.32464 \\
\hline 0.29774 & 0.33083 & 0 & 0.06494 & 0.02597 & 0.06494 & 0.12987 & 0.42857 & 74.4881 \\
\hline 0.02 & 0.18 & 0.2 & 0.148148 & 0.111111 & 0 & 0.074074 & 0.333333 & 73.04878 \\
\hline 0.257143 & 0.146939 & 0.110204 & 0.030612 & 0.091837 & 0.071429 & 0.091837 & 0.428571 & 72.01883 \\
\hline 0.018045 & 0.162406 & 0.162406 & 0.029557 & 0.098522 & 0.068966 & 0.08867 & 0.428571 & 71.12739 \\
\hline 0.114286 & 0.571429 & 0 & 0.171429 & 0.07619 & 0 & 0.038095 & 0.428571 & 68.42125 \\
\hline 0 & 0.866667 & 0 & 0 & 0 & 0.1875 & 0.145833 & 0.333333 & 68.26035 \\
\hline 0.433333 & 0 & 0.433333 & 0.2 & 0.066667 & 0 & 0.066667 & 0.333333 & 66.287 \\
\hline 0.330159 & 0.330159 & 0.08254 & 0 & 0.064935 & 0.12987 & 0.090909 & 0.428571 & 66.18268 \\
\hline 0 & 0.311111 & 0.088889 & 0.2 & 0.066667 & 0 & 0.066667 & 0.333333 & 65.98755 \\
\hline 0.02807 & 0.252632 & 0.252632 & 0.047619 & 0.071429 & 0 & 0.214286 & 0.333333 & 64.51453 \\
\hline 0.12 & 0.068571 & 0.154286 & 0.051948 & 0.064935 & 0.051948 & 0.116883 & 0.428571 & 64.21719 \\
\hline 0.08 & 0.28 & 0.04 & 0.185185 & 0 & 0 & 0.148148 & 0.333333 & 62.67215 \\
\hline 0.072727 & 0 & 0.327273 & 0 & 0.166667 & 0 & 0.166667 & 0.333333 & 61.9232 \\
\hline
\end{tabular}




\begin{tabular}{|c|c|c|c|c|c|c|c|c|}
\hline 0.65 & 0.108333 & 0.108333 & 0 & 0.075758 & 0.151515 & 0.106061 & 0.333333 & 61.18461 \\
\hline 0 & 0.311111 & 0.088889 & 0.222222 & 0.055556 & 0 & 0.055556 & 0.333333 & 60.97296 \\
\hline 0.133333 & 0.171429 & 0.038095 & 0.061224 & 0.102041 & 0 & 0.122449 & 0.428571 & 60.85272 \\
\hline 0 & 0.042424 & 0.424242 & 0.190476 & 0 & 0 & 0.142857 & 0.333333 & 60.36817 \\
\hline 0.366667 & 0.052381 & 0.314286 & 0.230769 & 0.076923 & 0 & 0.025641 & 0.333333 & 60.04174 \\
\hline 0.347368 & 0.385965 & 0 & 0.079365 & 0.031746 & 0.095238 & 0.126984 & 0.333333 & 58.51747 \\
\hline 0.285714 & 0.285714 & 0 & 0.103896 & 0.064935 & 0.051948 & 0.064935 & 0.428571 & 58.26666 \\
\hline 0.268908 & 0.10084 & 0.201681 & 0.034286 & 0.114286 & 0.08 & 0.057143 & 0.428571 & 57.78555 \\
\hline 0.2 & 0.2 & 0 & 0.079365 & 0.095238 & 0 & 0.15873 & 0.333333 & 57.46786 \\
\hline 0.130612 & 0.065306 & 0.146939 & 0.175824 & 0 & 0.043956 & 0.065934 & 0.428571 & 57.29696 \\
\hline 0.304762 & 0 & 0.152381 & 0.031746 & 0.063492 & 0.126984 & 0.063492 & 0.428571 & 54.75444 \\
\hline 0.048485 & 0 & 0.484848 & 0.133333 & 0 & 0 & 0.2 & 0.333333 & 53.61301 \\
\hline 0 & 0 & 0.571429 & 0.029557 & 0.098522 & 0.068966 & 0.08867 & 0.428571 & 53.31768 \\
\hline 0.017143 & 0.171429 & 0.154286 & 0.142857 & 0.107143 & 0 & 0.035714 & 0.428571 & 52.33126 \\
\hline 0.419048 & 0 & 0.209524 & 0.064935 & 0.025974 & 0.064935 & 0.12987 & 0.428571 & 52.06064 \\
\hline 0 & 0 & 0.466667 & 0 & 0 & 0.230769 & 0.102564 & 0.333333 & 51.92239 \\
\hline 0 & 0 & 0.666667 & 0.083333 & 0.0625 & 0 & 0.1875 & 0.333333 & 51.20401 \\
\hline 0.426667 & 0.106667 & 0 & 0.083333 & 0.125 & 0 & 0.125 & 0.333333 & 50.62276 \\
\hline 0.2 & 0.2 & 0 & 0.087719 & 0.105263 & 0.105263 & 0.035088 & 0.333333 & 50.51908 \\
\hline 0 & 0.742857 & 0 & 0 & 0 & 0.214286 & 0.071429 & 0.428571 & 50.48131 \\
\hline 0.123077 & 0 & 0.410256 & 0.133333 & 0 & 0 & 0.2 & 0.333333 & 50.4807 \\
\hline 0.12 & 0.068571 & 0.154286 & 0.142857 & 0.107143 & 0 & 0.035714 & 0.428571 & 48.98349 \\
\hline 0.2 & 0.2 & 0 & 0.079365 & 0.095238 & 0 & 0.15873 & 0.333333 & 48.34827 \\
\hline 0 & 0.8 & 0 & 0.041667 & 0 & 0.25 & 0.041667 & 0.333333 & 47.85062 \\
\hline 0 & 0 & 0.571429 & 0.07619 & 0.066667 & 0.07619 & 0.066667 & 0.428571 & 47.66561 \\
\hline 0.14359 & 0.184615 & 0.205128 & 0.133333 & 0 & 0 & 0.2 & 0.333333 & 47.60817 \\
\hline 0.457143 & 0 & 0 & 0.171429 & 0.07619 & 0 & 0.038095 & 0.428571 & 43.59197 \\
\hline 0 & 0.355556 & 0.044444 & 0.277778 & 0.027778 & 0 & 0.027778 & 0.333333 & 43.53208 \\
\hline 0.533333 & 0 & 0.333333 & 0.105263 & 0 & 0.105263 & 0.122807 & 0.333333 & 40.22468 \\
\hline 0.177778 & 0 & 0.355556 & 0.122807 & 0 & 0.157895 & 0.052632 & 0.333333 & 39.57291 \\
\hline 0.495238 & 0.247619 & 0 & 0 & 0.084034 & 0.151261 & 0.05042 & 0.428571 & 37.15172 \\
\hline 0.533333 & 0 & 0.333333 & 0 & 0 & 0.166667 & 0.166667 & 0.333333 & 36.3318 \\
\hline
\end{tabular}




$\begin{array}{rrrrrrrrr}0.533333 & 0 & 0 & 0.083333 & 0.125 & 0 & 0.125 & 0.333333 & 33.84496 \\ 0 & 0 & 0.342857 & 0 & 0 & 0.214286 & 0.071429 & 0.428571 & 33.45984 \\ 0.415584 & 0 & 0.155844 & 0 & 0 & 0.114286 & 0.171429 & 0.428571 & 31.73201 \\ 0.45 & 0 & 0.15 & 0.140351 & 0.035088 & 0 & 0.157895 & 0.333333 & 27.36593 \\ 0.457143 & 0 & 0 & 0.025974 & 0 & 0 & 0.25974 & 0.428571 & 25.54157\end{array}$

\section{$5^{\text {th }}$ Generation}

\begin{tabular}{|c|c|c|c|c|c|c|c|c|}
\hline $\mathrm{Li}$ & $\mathrm{Na}$ & $\mathrm{K}$ & Y & $\mathrm{Gd}$ & $\mathrm{La}$ & $\mathrm{Eu}$ & $\mathrm{Ti}$ & Luminance \\
\hline 0.032 & 0.368 & 0 & 0.2 & 0.067 & 0 & 0.07 & 0.333 & 115.0084 \\
\hline 0 & 0.4 & 0 & 0.19 & 0 & 0 & 0.143 & 0.333 & 88.7902 \\
\hline 0.04444 & 0.28889 & 0 & 0.2 & 0.0333 & 0 & 0.1 & 0.333 & 83.6275 \\
\hline 0.19649 & 0.2526 & 0.0842 & 0.233 & 0 & 0 & 0.1 & 0.333 & 81.4507 \\
\hline 0.02807 & 0.28 & 0.0253 & 0.133 & 0 & 0 & 0.2 & 0.333 & 80.7002 \\
\hline 0 & 0 & 0.4 & 0.095 & 0 & 0 & 0.19 & 0.429 & 80.3720 \\
\hline 0.08 & 0.28 & 0.04 & 0.145 & 0 & 0.1304 & 0.058 & 0.333 & 78.2063 \\
\hline 0.12308 & 0.15824 & 0.1758 & 0.031 & 0.0918 & 0.0714 & 0.092 & 0.429 & 76.9635 \\
\hline 0.06857 & 0.24 & 0.0343 & 0.159 & 0 & 0 & 0.127 & 0.429 & 74.9124 \\
\hline 0.01805 & 0.16241 & 0.1624 & 0.032 & 0.1058 & 0.0741 & 0.074 & 0.429 & 73.1792 \\
\hline 0.65 & 0.10833 & 0.1083 & 0.2 & 0.0667 & 0 & 0.067 & 0.333 & 72.5965 \\
\hline 0.33016 & 0.33016 & 0.0825 & 0 & 0.0595 & 0.119 & 0.107 & 0.429 & 69.4756 \\
\hline 0 & 0.04242 & 0.4242 & 0.076 & 0.0909 & 0 & 0.167 & 0.333 & 68.8037 \\
\hline 0.08889 & 0 & 0.4444 & 0.133 & 0 & 0 & 0.2 & 0.333 & 68.4475 \\
\hline 0.43333 & 0 & 0.4333 & 0 & 0.0758 & 0.1515 & 0.106 & 0.333 & 67.7259 \\
\hline 0.02105 & 0.18947 & 0.1895 & 0.034 & 0.1149 & 0.0805 & 0.103 & 0.333 & 67.6320 \\
\hline 0.12308 & 0.15824 & 0.1758 & 0.19 & 0 & 0 & 0.095 & 0.429 & 66.1652 \\
\hline 0.20317 & 0.20317 & 0.0508 & 0.045 & 0.0602 & 0.1203 & 0.06 & 0.429 & 65.8758 \\
\hline 0.26891 & 0.10084 & 0.2017 & 0.031 & 0.0918 & 0.0714 & 0.092 & 0.429 & 65.8038 \\
\hline 0.74286 & 0 & 0.1238 & 0 & 0.0758 & 0.1515 & 0.106 & 0.333 & 65.5487 \\
\hline 0.25714 & 0.14694 & 0.1102 & 0.034 & 0.1143 & 0.08 & 0.057 & 0.429 & 65.3775 \\
\hline 0.01805 & 0.16241 & 0.1624 & 0.032 & 0.1058 & 0.0741 & 0.074 & 0.429 & 65.3165 \\
\hline 0 & 0.68571 & 0 & 0.022 & 0.1099 & 0 & 0.154 & 0.429 & 64.9394 \\
\hline 0.01667 & 0.16667 & 0.15 & 0.133 & 0.1 & 0 & 0.1 & 0.333 & 63.4000 \\
\hline 0.39 & 0.43333 & 0.0433 & 0 & 0.0758 & 0.1515 & 0.106 & 0.333 & 62.8015 \\
\hline 0.49524 & 0 & 0.2476 & 0 & 0.0649 & 0.1299 & 0.091 & 0.429 & 62.1787 \\
\hline 0.04848 & 0 & 0.4848 & 0.044 & 0.0667 & 0 & 0.222 & 0.333 & 62.0066 \\
\hline
\end{tabular}




\begin{tabular}{|c|c|c|c|c|c|c|c|c|}
\hline 0 & 0 & 0.4 & 0.057 & 0.0286 & 0.0714 & 0.129 & 0.429 & 60.0690 \\
\hline 0 & 0.53333 & 0.1524 & 0.171 & 0.0762 & 0 & 0.038 & 0.429 & 59.5882 \\
\hline 0 & 0 & 0.3429 & 0.143 & 0.1071 & 0 & 0.036 & 0.429 & 59.2953 \\
\hline 0.13061 & 0.06531 & 0.1469 & 0.065 & 0.026 & 0.0649 & 0.13 & 0.429 & 59.1026 \\
\hline 0.12 & 0.06857 & 0.1543 & 0.176 & 0 & 0.044 & 0.066 & 0.429 & 58.9666 \\
\hline 0.08333 & 0 & 0.25 & 0 & 0.1667 & 0 & 0.167 & 0.333 & 58.1815 \\
\hline 0.26891 & 0.10084 & 0.2017 & 0.036 & 0.119 & 0.0833 & 0.048 & 0.429 & 57.8552 \\
\hline 0.30476 & 0 & 0.1524 & 0.03 & 0.0602 & 0.1203 & 0.075 & 0.429 & 56.8563 \\
\hline 0 & 0.31111 & 0.0889 & 0.182 & 0.0606 & 0 & 0.091 & 0.333 & 56.5314 \\
\hline 0.01905 & 0.19048 & 0.1905 & 0.11 & 0 & 0 & 0.176 & 0.429 & 55.9362 \\
\hline 0 & 0.46667 & 0 & 0 & 0 & 0.2381 & 0.095 & 0.333 & 55.2474 \\
\hline 0.19649 & 0.25263 & 0.0842 & 0.079 & 0.0952 & 0 & 0.159 & 0.333 & 54.6755 \\
\hline 0 & 0 & 0.5714 & 0.071 & 0.0625 & 0.0714 & 0.08 & 0.429 & 54.2594 \\
\hline 0.24381 & 0.1219 & 0.0914 & 0.031 & 0.0918 & 0.0714 & 0.092 & 0.429 & 54.1867 \\
\hline 0.08333 & 0 & 0.5833 & 0.099 & 0.0864 & 0.0988 & 0.049 & 0.333 & 53.5214 \\
\hline 0.2 & 0.2 & 0 & 0.212 & 0 & 0 & 0.121 & 0.333 & 49.6609 \\
\hline 0.53878 & 0.0898 & 0 & 0.065 & 0.026 & 0.0649 & 0.13 & 0.429 & 49.6097 \\
\hline 0.41905 & 0 & 0.2095 & 0.163 & 0.0204 & 0.0408 & 0.061 & 0.429 & 47.6601 \\
\hline 0.13333 & 0.23333 & 0.0333 & 0.185 & 0 & 0 & 0.148 & 0.333 & 46.4258 \\
\hline 0 & 0 & 0.8 & 0.042 & 0 & 0.25 & 0.042 & 0.333 & 41.9618 \\
\hline 0.13333 & 0.26667 & 0 & 0.083 & 0.1 & 0 & 0.15 & 0.333 & 41.8646 \\
\hline 0 & 0 & 0.4 & 0 & 0 & 0.1524 & 0.133 & 0.429 & 39.4922 \\
\hline 0.41905 & 0 & 0.2095 & 0.11 & 0 & 0 & 0.176 & 0.429 & 38.2298 \\
\hline 0.17778 & 0 & 0.3556 & 0.137 & 0 & 0.1765 & 0.02 & 0.333 & 35.8060 \\
\hline 0.17778 & 0 & 0.3556 & 0.156 & 0 & 0 & 0.178 & 0.333 & 30.5994 \\
\hline 0.3 & 0.17143 & 0.1286 & 0.133 & 0 & 0 & 0.2 & 0.333 & 28.6716 \\
\hline 0.15556 & 0.2 & 0.0444 & 0.111 & 0 & 0.2222 & 0 & 0.333 & 24.6570 \\
\hline
\end{tabular}




\section{Confirmatory Experiment based on conventional Solid State Reaction}

Synthesis

\begin{tabular}{|c|c|}
\hline Raw Mate & \\
\hline $\mathrm{Eu}_{2} \mathrm{O}_{3}$ & Kojundo $(99.99 \square)$ \\
\hline $\mathrm{Y}_{2} \mathrm{O}_{3}$ & Kojundo $(99.99 \square)$ \\
\hline $\mathrm{Gd}_{2} \mathrm{O}_{3}$ & Kojundo $(99.99 \square)$ \\
\hline $\mathrm{TiO}_{2}$ & Aldrich (99.99 $\square)$ \\
\hline $\mathrm{Na}_{2} \mathrm{CO}_{3}$ & Kojundo (99.9ロ) \\
\hline
\end{tabular}

The raw materials were blended completely with a certain amount of acetone, dried, and successively fired in a two-step process, i.e., $900{ }^{\circ} \mathrm{C}$ for 2 hours and finally $1100{ }^{\circ} \mathrm{C}$ for 2 hours in an ambient atmosphere. Intermediate pulverizations were also done during the two-step process.

The experimental results were graphed as shown below;

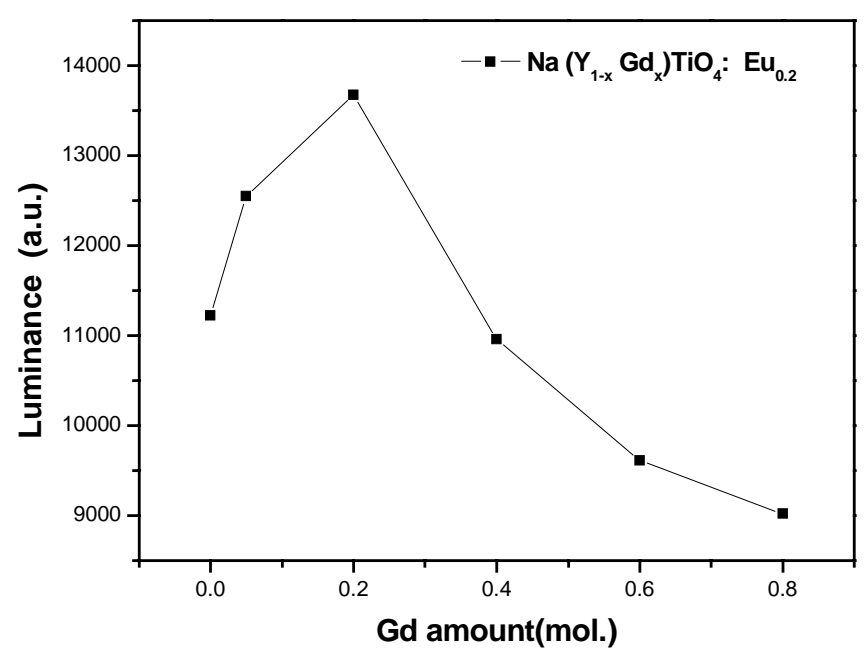



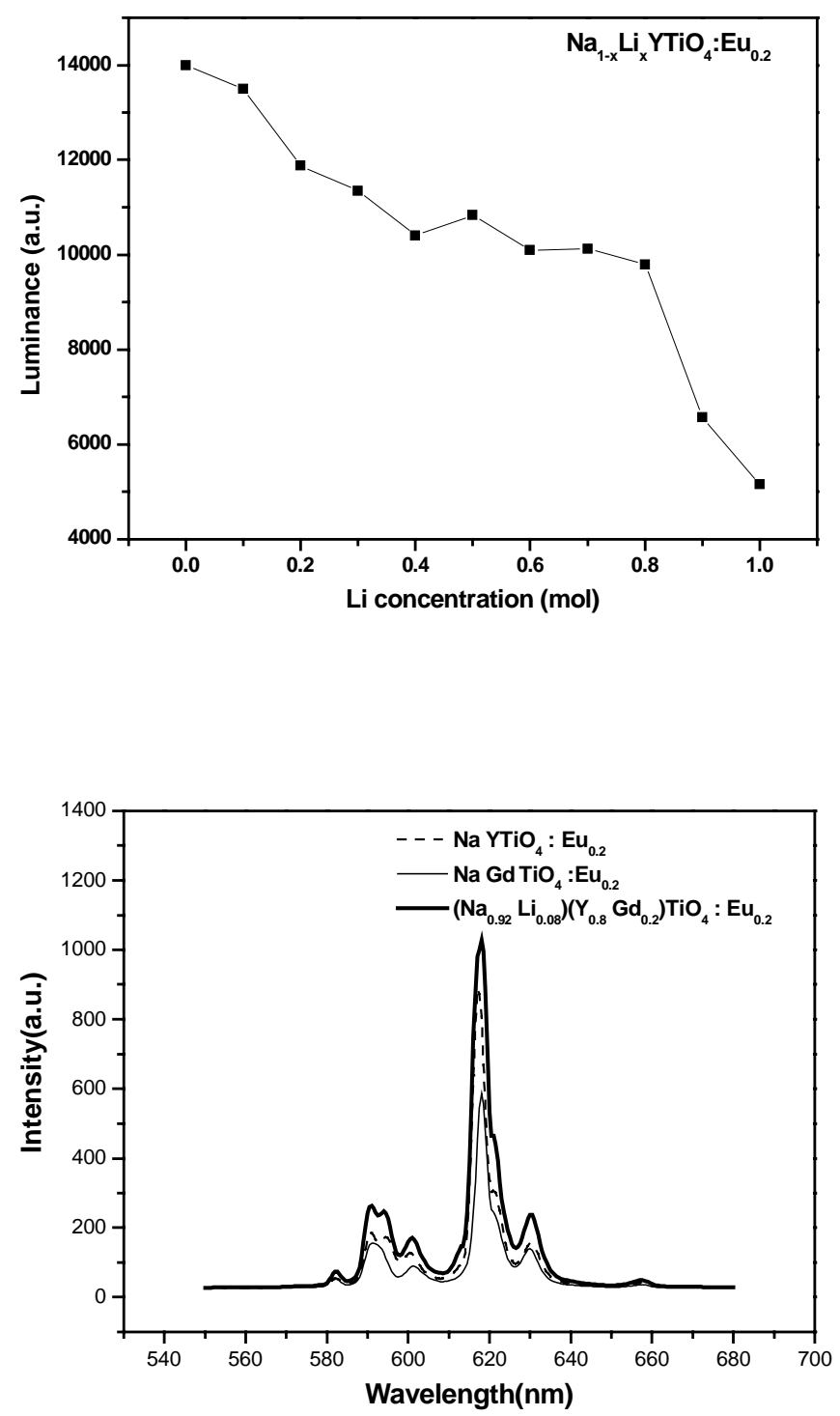